\title{
No Laws and (Thin) Powers In, No (Governing) Laws Out
}

\author{
Stavros Ioannidis ${ }^{1}$, Vassilis Livanios ${ }^{2} \&$ Stathis Psillos $^{3}$
}

${ }^{1}$ Corresponding author, $\mathrm{MA}, \mathrm{PhD}$, Postdoctoral researcher, Dept of History and Philosophy of Science, National and Kapodistrian University of Athens, University Campus, 15771 Athens, Greece; email: sioannidis@phs.uoa.gr

${ }^{2} \mathrm{MSc}$, PhD, Assistant Professor, Dept of Classics and Philosophy, University of Cyprus, Eressou 1 \& Kallipoleos, P.0. Box: 20537, 1678 Nicosia, Cyprus; email: livanios.vasileios@ucy.ac.cy

${ }^{3} \mathrm{MSc}$, PhD, Professor of Philosophy of Science and Metaphysics, Dept of History and Philosophy of Science, National and Kapodistrian University of Athens, University Campus, 15771 Athens, Greece; email:psillos@phs.uoa.gr

\begin{abstract}
Non-Humean accounts of the metaphysics of nature posit either laws or powers in order to account for natural necessity and world-order. We argue that such monistic views face fundamental problems. On the one hand, neo-Aristotelians cannot give unproblematic powerbased accounts of the functional laws among quantities offered by physical theories, as well as of the place of conservation laws and symmetries in a lawless ontology; in order to capture these characteristics, commitment to governing laws is indispensable. On the other hand, ontologies that entirely exclude some kind of power ascription to worldly entities (such as primitivism) face what we call the Governing Problem: such ontologies do not have the resources to give an adequate account of how laws play their governing role. We propose a novel dualist model, which, we argue, has the resources to solve the difficulties encountered by its two dominant competitors, without inheriting the problems of either view. According to the dualist model, both laws and powers (suitably conceived) are equally fundamental and irreducible to each other, and both are needed in order to give a satisfactory account of the nomological structure of the world. The dualist model constitutes thus a promising alternative to current monistic views in the metaphysics of science.
\end{abstract}

\section{Introduction}

For many philosophers, certainly in the seventeenth century but also in the latter half of the twentieth, laws underpin the causal fabric of the world and ground and explain the regularity there is in it. They capture a sense of necessity or inevitability in the workings of worldly entities. They are thus being granted modal force; it is this modal force which gives the laws of nature their explanatory power. Laws are relied upon not only to explain (and predict) what actually happens, but also to ground what could or could not have happened (by supporting relevant counterfactual conditionals). ${ }^{1}$

In the recent literature in philosophy of science, however, there is a call to 'dethrone'

\footnotetext{
1 This tradition goes back to Descartes (1982). For a detailed account of this tradition and its relation to the earlier dominant Aristotelianism, see Psillos (2018).

2 We think, but will not argue for this now, that the origins of this dualist model can be found in Leibniz's mature (post 1680) work and especially in his critique of occasionalism, which posited laws but divested matter of all power. For some preliminary discussion, see Psillos (2018).
} 
laws as the source of natural necessity. Two are the dominant tendencies: either to eliminate laws altogether from scientific ontology as a relic of a bygone era, thereby denying them any role in the workings of nature (Cartwright \& Ward 2016); or to grant them an anemic status as mere side-effects (or summaries) of the causal powers of things, with laws playing no distinctive role of their own (Bird 2007). Either way, on offer is a lawless metaphysics of nature, with causal powers occupying the place and roles that laws of nature used to play according to the traditional view. The conception of a law-governed world is thus eliminated in favour of the Aristotelian idea that activity and change in the world are grounded in the natural causal powers of worldly entities (cf. Ellis 2001; Mumford 2004; Bird 2007; Marmodoro 2010; Mumford \& Anjum 2011; 2018).

In this paper we will argue that the lawless power-based ontology offered by neoAristotelians faces a serious problem: a power-based ontology that posits no nomological connections among powers, does not suffice to capture central aspects of laws as revealed by physical theories; in particular, neo-Aristotelians have great trouble giving power-based accounts of the functional laws among quantities offered by physical theories, of the place of conservation laws and symmetries in a lawless ontology, as well as the role of counterlegals in scientific theorising. We will argue that, in order to capture these characteristics, laws that govern (powers) must have a place within a naturalistic ontology. We will go on to claim, however, that ontologies which do away with some kind of power ascription to worldly entities, for instance primitivism about laws, do not have the resources to give an adequate account of how laws play their governing role; to do that, giving back some power to worldly entities is inevitable. Hence, we battle in two fronts: we argue against the powerful-but-lawless account of neo-Aristotelians as well as against the lawful-but-powerless account of views such as primitivism. On the positive side, we will advance and defend a novel dualist model, according to which positing both laws and some kind of power is necessary for an adequate account of the nomological structure of the world, according to which laws govern the behaviour of worldly things and worldly things can execute the laws. ${ }^{2} \mathrm{Hence}$, though this dualist model will highlight the limitations of a power-based ontology, it will show, that there is a role for powers, suitably understood, to play within the metaphysics of science.

Here is the road map. We will start with some preliminary remarks that will set the stage for what follows (section 2). Next (section 3), we will present our main argument against neo-Aristotelianism and lawless ontologies in general. In section 4, we will present the main problem, the Governing Problem, that, we think, affects ontologies that posit governing laws but no powers. Then, in section 5 , we will present the general contours of our dualist model that admits of both powers and governing laws and defend it against criticism. Finally, in section 6 we will compare our dualist model with three recent views that give a role to both laws and powers and argue that our dualist model is to be preferred.

\section{Powerful Lawlessness vs Lawful Powerlessness}

Not all philosophers agree that there is necessity in nature. Typically, neo-Humean philosophers deny that the regularity there is in the world is the result of necessary connections among distinct existents, e.g., properties of particulars. But among those

2 We think, but will not argue for this now, that the origins of this dualist model can be found in Leibniz's mature (post 1680) work and especially in his critique of occasionalism, which posited laws but divested matter of all power. For some preliminary discussion, see Psillos (2018). 
who take it that some kind of necessity renders the world regular, there is a fundamental split regarding how best to answer the question: what is the source of natural necessity? There are those who take it that necessary connections are grounded in the properties of worldly entities conceived of as powers and those who take it that they are grounded in laws, conceived of as governing the properties and the particulars thus related.

\subsection{Powerful Lawlessness}

Those philosophers who posit irreducible powers (aka purely dispositional properties) take it that powers have modal force: they are modally-laden properties. As such, they are causally active entities, standing in definite relations of entailment, exclusion and production with other powers. The identity of properties is not fixed by any individuating factor such as a quiddity; rather it is fixed by the network of causal relations to other properties; and hence by each power's causal role. On this view, powers fix completely the nomic structure of the world, the implication being that laws are nothing over and above the relations among powers. Some advocates of this view (e.g., Mumford) take it that, a fortiori, laws are nothing: there are no laws. Others, e.g., Bird, take it that laws flow directly from powers. Consequently, laws are real but not fundamental to our ontology because they are fixed by powers, where, for Bird at least, the fixing is supervenience: "laws are general relations among properties that supervene on potencies" $(2007,200)$. At the same time, "laws are no addition of being beyond potencies" $(2007,201)$. Laws fall in the category of "supervening or nonfundamental natural entities" whereas potencies are "fundamental natural entities" (2007, 202).

The dominant view among the friends of powers is dispositional essentialism: properties have dispositional essences (hence, properties are individuated by their essences, which confer on their possessors certain powers) and laws are ontologically dependent on these essences. Mumford, by the way, agrees that the nature of properties is dispositional but denies that properties have essences; rather, their individuation is holistic (see his 2004, 123). We will call the view that puts the powers ahead of laws (with or without laws) Powerful Lawlessness ${ }^{3}$.

\subsection{Lawful Powerlessness}

Those philosophers who posit laws as the source of natural necessity take it that laws govern their instances. They are governing laws. What exactly governing amounts to is a controversial issue, but it involves at least a) entailment of the corresponding regularity and b) modal force: laws ground various relevant counterfactuals such as if there had been an extra X, it would have been Y. Typically, governing laws are taken to determine the corresponding regularity (the regularity would not be there, had the law not been there) and hence to determine the course of events occurring in the world. The so-called ADT-approach (defended independently by Dretske (1977), Tooley (1977) and

\footnotetext{
${ }^{3}$ We take Powerful Lawlessness to include any view that posits powers as fundamental and laws as derivative (or non-existent), even if it adopts the existence of categorical features (e.g. spatiotemporal relations, cf. Molnar 2003, Ellis 2005). So, the problems Powerful Lawlessness faces and are presented in this paper emerge both within a dispositional monistic framework with 'thick' powers (which are powers whose essences are 'sensitive' to categorical features because they have 'absorbed' the dependence of the strength of power's action on spatiotemporal relations), as well as within property-dualistic frameworks, as long as a) the categorical properties are not fundamental and their role is only, as Ellis (2005) says, to set the stage for the action of powers and b) laws just describe the action of powers. We discuss the relationship between powers and spatiotemporal relations further in section 3.1.
} 
Armstrong (1983)) is a characteristic example of the governing conception. The core of this view is that laws are contingent necessitating relations among universals. Far from being the law, the regularity 'All Fs are Gs' holds in the world because the law N(F, G) holds and yields it, where $\mathrm{N}$ is a second-order relation among universals F-ness and Gness which somehow enforces that whenever F-ness is instantiated so is G-ness.

Notably, the properties that stand in relation $\mathrm{N}$ to each other are categorical and hence non-modal.

The recently popular primitivist account of lawhood is another governing conception. Primitivists take it that laws are real, but ultimate and primitive constituents of reality, which hold with irreducible necessity and guide (and hence govern) the kinematical and dynamical behaviour of worldly entities (Carroll 1994; Maudlin 2007). As Maudlin put it: "laws of nature stand in no need of 'philosophical analysis'; they ought to be posited as ontological bedrock" $(2007,1)$. Despite the fact that primitivists are not clear on the relation between laws and properties, it is correct to say that their conception is governing, since the fundamental laws of temporal evolution, for example, produce (and explain) whatever regularity there is in the world. Given that all these accounts prioritise laws over properties and deny a role to powers, we will call them, (collectively) Lawful Powerlessness.

\subsection{Against Monistic Views}

Both conceptions, it should be noted, admit the existence of natural necessity, but locate its source differently. On the Powerful Lawlessness (PL) view, necessity is internal: necessary relations supervene on the intrinsic properties of the related particulars. It's part of the basic metaphysical structure of the universe: the nature of properties qua powers. On the Lawful Powerlessness (LP) view, these relations of necessitation are external in that they do not supervene on the intrinsic properties of the related universals; they are a (contingent) add-on. Still on both accounts, but for different reasons, regularities are enforced on the world: when one property is instantiated the other has to be instantiated too.

It should be stressed that both of the currently popular views about the fundamental structure of the world are monistic. It has been a general presupposition in the current literature that appealing either only to modal properties or only to laws is sufficient to provide a) an adequate account of the source of natural necessity and b) a plausible metaphysical explanation of the world order. In the sequel, we will argue that both monistic approaches (PL and LP) are problematic. This is our main motivation to develop a novel, dualistic account in which both powers and laws (properly analysed) are indispensable parts for an adequate metaphysical explanation of natural necessity and world order. Before delineating the basic tenets of our own view, let us review the reasons to think that both monistic approaches are inadequate.

\section{Powers Without Laws?}

\subsection{Problems with the Derivation}

The first question for the defenders of Powerful Lawlessness is the following: can they go beyond the slogan that laws 'flow' from powers and offer a more detailed account of how laws are supposed to be so derived? Bird $(2007,46)$ aims to provide such an account by deriving a lawful regularity from the dispositional essences of properties. According to Bird, if $\mathrm{P}$ is a fundamental modal property (a potency in Bird's terminology) then it is essentially and hence necessarily associated with a disposition to yield a particular manifestation $\mathrm{M}$ in response to a particular stimulus S. Hence, 
$\left(\mathrm{CA}_{\square}\right) \square\left(\mathrm{D}_{(\mathrm{S}, \mathrm{M})} \mathrm{X} \leftrightarrow \mathrm{Sx} \square \rightarrow \mathrm{Mx}\right)$, where CA is the acronym of 'conditional analysis' and $\mathrm{D}_{(\mathrm{S}, \mathrm{M})} \mathrm{x}$ ' symbolises ' $\mathrm{x}$ is disposed to manifest $\mathrm{M}$ in response to stimulus $\mathrm{S}$ '.

Being a potency, property $P$ is such that

$\left(\mathrm{DE}_{\mathrm{P}}\right) \square\left(\mathrm{Px} \rightarrow \mathrm{D}_{(\mathrm{S}, \mathrm{M}) \mathrm{x}}\right)$

That is, the real essence of $\mathrm{P}$ is its power to give characteristic manifestation $\mathrm{M}$ under stimulus conditions $\mathrm{S}$.

Now, $\left(\mathrm{CA}_{\square}\right)$ and $(\mathrm{DE})$ ) yield

$$
\square(\mathrm{Px} \rightarrow(\mathrm{Sx} \square \rightarrow \mathrm{Mx}))
$$

Assume a world $\mathrm{w}$ in which some object $\mathrm{x}$ possesses $\mathrm{P}$ and in which $\mathrm{x}$ also acquires stimulus S. In w

(II) $\quad \mathrm{Px} \& \mathrm{Sx}$.

(I) and (II) yield

(III) $\mathrm{Mx}$

Hence,

Hence,

$$
(\mathrm{IV})(\mathrm{Px} \& \mathrm{Sx}) \rightarrow \mathrm{Mx}
$$

$$
\text { (V) } \forall \mathrm{x}((\mathrm{Px} \& \mathrm{Sx}) \rightarrow \mathrm{Mx})
$$

That's a neat derivation of a qualitative generalisation. Being thus, it fails to show how quantitative-mathematical relations among properties 'flow' from their dispositional essences. (V) above states that the property $\mathrm{P}$ and the stimulus $\mathrm{S}$ lead to the manifestation $\mathrm{M}$, but it does not necessarily capture the relation among the three elements. In particular, it does not capture the quantitative relation between the property (qua quantity) and its manifestation. Hence, as Vetter $(2012,209-15)$ pointed out, Bird's derivation cannot actually describe physics' functional laws among quantities. As an example, she referred to Coulomb's law for the interaction of point charged particles. Here is how Vetter $(2009,325)$ put the point: "Like most (all?) fundamental laws of physics, Coulomb's Law states not merely a relation between properties, but a very special kind of relation - namely, a mathematical function between properties of a very special kind - namely, quantities. (V), on the other hand, states a much simpler, non-mathematical relation between properties that look rather like qualities". Could the advocate of dispositional essentialism argue that powers are (fully) determinate? That is, that Coulomb's law refers to the power of charge of specific value $Q$ to attract or repel other charges of specific values? The 'law' then would be: Everything that has charge $Q$ thus-and-so and is in specific distance $r$ will manifest a specific force F. But this is not Coulomb's law. It is totally unclear what exactly all similar instances have in common in virtue of which they could be taken to be instances of Coulomb's law. 
Vetter suggests an alternative formulation of the characterisation of charge that is open to the dispositional essentialist:

(I+) $\square$ (x has charge e $\rightarrow \forall$ charges $\mathrm{q}_{\mathrm{i}} \forall$ distances $\mathrm{r}_{\mathrm{i}}$ (x is at $\mathrm{r}_{\mathrm{i}}$ from $\mathrm{q}_{\mathrm{i}} \square \rightarrow \mathrm{x}$ exerts force $\mathrm{F}_{\mathrm{i}}=\varepsilon \frac{\mathrm{e} q_{i}}{r_{i}^{2}}$ )

This reformulation, as Vetter notes, differs from Bird's initial formulation (I) in that the stimulus and the manifestation of the disposition cannot be characterised independently of each other: if we specify the manifestation as the force given by Coulomb's law, we have to specify $\mathrm{q}_{\mathrm{i}}$ and $\mathrm{r}_{\mathrm{i}}$, which however are part of the stimulus. Vetter's suggestion is to understand (I+) in terms of a manifestation only: 'the exertion of a force that stands in a certain correlation to certain features of the environment' (327).

While (I+) has the advantage over (I) that it can be used to derive Coulomb's law, rather than instances of Coulomb's law (as in Bird's case), there is a crucial problem with both derivations, and, we submit, with all similar kinds of proposed derivation of Coulomb's law as well as other laws in physics that specify relations among quantities. As Cartwright and Pemberton (2013) have noted, dispositional essentialists are committed to the view that (at least some) powers have a 'dense' causal profile, that is that the power has it within itself that it will manifest itself in a certain concrete way for each and every value of its manifestation partners, where this set of values may be infinite. So for instance, if the electrostatic force $F_{i}=\varepsilon \frac{q_{j} q_{i}}{r_{i}^{2}}$ is the manifestation of charges given the inverse square of the distance between them, as in Coulomb's law, then the power of the charge $q_{i}$ to attract or repel other charges $q_{j}$ is fixed for all values of $\mathrm{q}_{\mathrm{i}}$ and $\mathrm{q}_{\mathrm{j}}$ and all the values $\mathrm{r}_{\mathrm{i}}$ of the distance between the charges, where $\mathrm{r}_{\mathrm{i}}$ takes its value from the interval $(0, \infty)$. So, even if adoption of $(\mathrm{I}+)$ allows Coulomb's law to be derived and shows how Coulomb's law is fixed by the power of the charge, the price to pay would be quite steep, as such a view of powers is unnecessarily complex.

Cartwright and Pemberton's own suggestion is to think of the manifestation of a power in terms of a 'canonical effect' or a 'contribution' that the power makes in different circumstances. Such a view has the advantage of avoiding the complexity of the 'causal profile' account. But when we consider how such a view treats a law that specifies relations among quantities, such as Coulomb's law, the problem remains: what determines what will happen in each different situation, i.e. how the various contributions of the powers will be combined, such as Coulomb's law will be satisfied? It would seem that we need laws of combination of the various contributions (for instance, the parallelogram-law for the addition of component forces or more complex tensorial laws) in order to explain the occurrence of specific effects. And it seems that these laws, like the conservation laws that we will discuss in the next section, cannot flow from the nature of powers. Thus, the 'contribution' view seems to strengthen our point for the indispensability of laws. ${ }^{4}$

\footnotetext{
${ }^{4}$ Cartwright and Pemberton acknowledge this, when they say: "[i]t might be objected that the account of powers we offer does not eliminate the need for laws of nature since it still leaves need for rules of combination that are independent of the powers in nature. So that unlike some other powers accounts, we may not have succeeded in getting governance back in to nature. We concede that this may be so. There may be ways to get rules for combining powers into nature itself in a way that fits a pure powers ontology and there may not. This remains work for the future" $(2013,94-95)$. Note also that our point that powers cannot account on their own for the nomological structure of the world holds, as far as we
} 
A case such as Coulomb's law, then, gives rise to the following questions. How does charge 'manage' the information that is available to it? How does it 'use' it so that Coulomb's law is satisfied? In other words, why does it follow from the fact that Coulomb's force is distance dependent that it is inverse-square-of-the-distance dependent? And how does the concrete charge happen to be sensitive to the right kind of information available in the inventory of possible values in such a way that Coulomb's law is satisfied? These are substantive questions which suggest the following answer: it is because the charge's behaviour is governed by Coulomb's law that the charge's power to attract and repel other charges is manifested in the way it does and not the other way around. As we shall show in the next section, a main problem with any alleged derivation of laws from properties is that the quantitative relations among properties do not rightaway follow from these properties being powers. More is needed, and in particular laws are needed (e.g., conservation laws) for such a derivation.

\subsection{Problems with Conservation Laws}

Let's move on, then, to a fundamental problem, in our view, that the advocates of PL face: can PL provide adequate metaphysical explanations of all nomic notions used by contemporary science in terms of modal properties and their nature? More precisely, can PL accommodate the cases of conservation laws, symmetries and global principles? Our answer is negative and in what follows we offer some of the main reasons that lead us to that conclusion (see also Livanios 2017).

Let us begin with conservation laws. It is a fact about our world that various quantities are conserved in all interactions; examples of such quantities are massenergy, electric charge, momentum, angular momentum. It is also well known that contemporary physical science strongly suggests that specific kinds of symmetries (the continuous ones) are intimately related to the conservation of these physical quantities. In particular, a famous theorem of Amalie Emmy Noether (1918) says that for each continuous symmetry of the Lagrangian function of a physical system there is a quantity which is conserved by its dynamics. The application of Noether's (first) theorem in the case of various continuous symmetries provides a unified and non-ad hoc explanation of the existence of conservation laws and conserved quantities in our world.

Conservation laws pose at least three significant challenges to PL. The first challenge is this. Take a specific conservation law, say the conservation of electric charge. This law, like all conservation laws, holds for closed systems of particles, not for individual particles. This leads to two problems for Bird's derivation. The first problem is that Bird's derivation of laws from dispositional essences is based on properties of individual particles such as charge; but the law of conservation of charge holds for a system of particles, rather than for individual particles. It seems then that Bird, and proponents of PL in general, face the problem of how to actually derive conservation laws from the essences of properties of individual things. The second problem is that conservation laws holds for closed systems of particles and the only closed system of particles is the whole world, not individual particles. To address the second problem, a natural maneuver of the advocates of PL is to offer an account that appeals to the nature of the whole world.

According to one version of this account (Bigelow et al. 1992), our world is the unique actual member of a natural kind whose real essence requires conservation of

can see, regardless of how one views the manifestation of a power in ontological terms (e.g. as an event, state of affairs or process). 
certain quantities; in other words, conservation laws 'flow' from the kind-essence of the actual world. Bigelow et al., most probably in order to avoid the accusation of ad hocness, appeal to the world-as-one-of-a-kind view to provide a covering explanation for the emergence of all laws of nature. They claim that, if conservation laws arise out of the essence of the whole world, and granting that conservation laws differ from the other ones only in scope, then other laws (concerning parts of the world) must also arise out of the world-essence. Moreover, they suggest that the essences of parts of the world, and the connections which depend on them, may both contribute to the worldessence; and only insofar as they do that, can laws of nature arise out of them (cf. 1992, 386-7). Yet, if that is true, the world-essence explanation is at least redundant as far as the case of non-conservation laws is concerned. Since Bigelow et al. have presumably already provided an explanation of non-conservation laws as emanating from particular kind-essences, what more (besides, of course, escaping the ad hoc-ness charge) can the global, coarse-grained, explanation in terms of the world-essence offer?

Another version of the view under consideration is provided by Bird (2007, 213-4). He suggests (without finally endorsing) that conservation laws could 'flow' from the dispositional essence of the property being a world which the actual world definitely instantiates. This metaphysical explanation, however, is either ad hoc or poor. It is ad hoc if the property being a world is posited only in order to explain conservation facts. For the actual world must have the property of being a world with conservation laws, that is a world like ours! But suppose that positing the property of being a world is meant to provide an explanation of all worldly facts. It would then be a poor explanation since it would not be fine-grained explanation of the various types of laws that obtain in the world.

The second challenge is this: how can the advocates of PL provide a plausible unified metaphysical explanation of the phenomenon of conservation in general if they were to reject (for the reasons given above) the accounts offered by Bigelow et al., and Bird? The problem is that conservation applies to many different physical properties that seem to have nothing in common except of the 'feature' of being a physical property. So, if we reject a world-nature that in a sense can unify the various conservation laws, it is not clear on what grounds (that is, on the nature of which property) can the advocates of PL provide a bottom-up explanation of the phenomenon of conserved quantities.

Lastly, there is a third, and more general, challenge that conservation laws pose for PL-ists. Consider an interaction between two charged particles. Since charge is conserved, the characterisation of such an interaction requires a relevant conservation law. So, given that the conservation law does not 'flow' from the essence of charge (which, as characterised by the dispositional essentialist is a property of individual particles), nor from the essence of the relevant natural kind (as Bigelow et al. acknowledge), the behaviour of the charged particles is grounded on two things: the dispositional essence of charge (assuming that it has one) and the law of conservation of charge. ${ }^{5}$

That's a key point. For whether or not we accept the further claim that conservation laws 'flow' from the world-essence, it follows forcefully that these laws have a governing

\footnotetext{
5 The intertwinement of interaction laws with conservation laws can be also brought to the fore by the approximate conservation of flavour quantum numbers of elementary particles. It is well known that quantum numbers such as strangeness, charm or beauty that characterise types of elementary particles are preserved under strong and electromagnetic interactions, but not under weak interaction. This fact clearly suggests that a complete characterisation of, say, strong interaction and its differences with weak interaction cannot be provided without reference to the conservation laws of those quantum numbers.
} 
function: it affects the nature of particular interactions of charged particles. This can only be an admission of defeat by the PL-ists: governing laws crop up again. The advocates of PL have to abandon one key tenet of their views, viz., that powers are the sole source of natural necessity and that therefore, there is no other entity over and above those powers, which is able to 'direct' powers (and hence objects) in what to do.

\subsection{Problems with Symmetries}

The case of fundamental symmetries is equally disturbing for PL. Symmetries posit extra constraints upon the behaviour of properties beyond those posited by their dispositional nature. This is at odds with PL. Actually, PL can be saved either by insisting that symmetry principles are merely epistemic features of the ways humans represent the world or by embracing the view that they are concerned with the essential properties of the kind of world we live in. The first option is adopted by Bird, who concludes that "the dispositional essentialist ought to regard symmetry principles as pseudo-laws" and that "symmetry principles and conservation laws will be eliminated as being features of our form of representation rather than features of the world requiring to be accommodated within our metaphysics" (Bird 2007, 214).

This first option would be plausible had the sole role of symmetry principles been to impose constraints upon the physical properties. However, that is not actually the case. The application of symmetry principles has a much wider scope, and that is a fact which in some cases grounds powerful arguments in favour of an ontological interpretation of symmetries - consider, for instance, the role of symmetry principles in the prediction of previously unknown elementary particles. ${ }^{6} \mathrm{~A}$ well-known example is the prediction of $\Omega^{-}$particle via the eightfold way, an organisational scheme for hadrons. This scheme is a consequence of flavor symmetries between various kinds of quarks and its application to the spin-3/2 baryons yields a classification in a form of a decuplet. Famously, one of the particles of this decuplet, $\Omega^{-}$, had never been previously observed when the eightfold way was proposed. This particle was actually observed in 1964 by a particle accelerator group at Brookhaven.

Now, the second option leads to the same difficulties as the case of conservation laws. Here again, the advocate of PL would argue that symmetries characterise the world as one-of-a-kind. By the same token, our reply will be similar too. If the advocates of PL give a power-based explanation of symmetries, such an explanation should have the following form: something governs or constrains the behaviour of properties. But this is in essence to buy into a 'governing' conception, thereby in effect abandoning PL.7

\subsection{Problems with Laws}

Insofar as they allow laws at all, the advocates of PL hold that all worlds with the same natural properties as the actual one, have the actual laws as well. So, for them, laws are metaphysically necessary. This kind of metaphysical necessity seems to be offered for free, since it follows from the criterion of identity of properties in combination with the claim that laws are just summaries of the causal profile of properties. Now, there are

\footnotetext{
${ }^{6}$ There is more that could be said about symmetries and conservation laws in relation to power ontologies; the purpose of sections 3.2 and 3.3 is to highlight some salient problems and sum up work that has been published elsewhere, which, for reasons of space, cannot be fully recited here. The interested reader should look at Chakravartty (2019), French (2014) and Livanios $(2010 ; 2018)$. 7 The existence of fundamental constants poses an additional significant threat to a power-based ontology. In the interest of space, we do not discuss this issue here but refer the reader to Livanios (2014), for a detailed account.
} 
powerful intuitions for the metaphysical contingency of laws. Be that as it may, the problem is that the alleged metaphysical necessity of laws stands in some cases in contrast to actual scientific practice. Scientists often appeal to counterfactuals having antecedents opposed to actual laws or counterfactuals having antecedents that contain laws in which fundamental constants (which current physics cannot fully determine) take on values that differ from the actual ones. But if there are no law-violating possibilities, then counterlegals will be vacuously true. It is, to say the least, not clear how vacuously true propositions can have any impact in actual scientific work. Given that the above do have an impact in scientists' thought, it seems that there is a tension here between PL's commitments and scientific practice. Hence, a burden on PL-ists is to develop an alternative account of the semantics of counterfactuals that secures that counterlegals are not vacuously true (see Ellis 1999; Handfield 2004).

To recap: the difficulties posed to Powerful Lawlessness (from the existence of functional laws among quantities, conservation laws, symmetries, and the function of counterlegals in scientific practice) show that not only is there no special need to metaphysically interpret basic scientific concepts in terms of sui generis modal properties but also that positing only them (at the expense of laws) is not enough to ground illuminating analyses of laws and principles that have an extremely significant role in science. Furthermore, it seems that laws, qua entities distinct from powers that are nonetheless able to govern them, are indispensable for the explanation of various facts concerning modal properties themselves, as will be explained more fully in the next sections.

\section{Laws Without Powers?}

We have seen that Powerful Lawlessness faces significant problems. Given the importance of conservation laws, symmetries, constants and functional laws in current physical theory, we have good reasons to accept laws of nature as fundamental items in our ontology, distinct from powers of things. Does this mean that we should embrace some form of Lawful Powerlessness and eliminate powers altogether? We are now going to argue that such powerless ontologies face a fundamental difficulty: without powers, it is not clear at all how governing laws can play their role.

\subsection{From the Inference Problem to the Governing Problem}

The Inference Problem, which was originally posed by van Fraassen (1989) and constitutes perhaps the most discussed difficulty faced by non-Humean accounts of laws of nature, is this. Any conception of laws must show how there is a valid inference from the laws there are to the regularities that hold in the world. Humean accounts of lawhood do not face this problem, since laws are, ultimately, regularities. But all those non-Humeans accounts which make laws distinct from regularities should show how regularities can be recovered. In other words, it should be shown how we get from 'It is a Law that P' to the relevant regularity. For instance, in the ADT-approach, how can an atomic second-order fact (e.g. the holding of the nomic necessitation $\mathrm{N}$ between universals $F$ and $G$ ) determine a first-order general fact (the regularity that for each object $\mathrm{x}$, if $\mathrm{x}$ is $\mathrm{F}$ then $\mathrm{x}$ is $\mathrm{G}$ )? Or take primitivism. If, as Maudlin says, "[l] aws are ontological primitives at least in that two worlds could differ in their laws but not in any observable respect" $(2007,17)$, how can we go from the laws there are in the world to the regularities?

This problem has been thoroughly discussed (see, for example Sider 1992, Armstrong 1993, Pages 2002, Hildebrand 2013, Schaffer 2016); nevertheless, in our 
view, there are still aspects of it that are largely unexplored. ${ }^{8}$ One of these aspects regards the fact that it is only by governing the properties that non-Humean laws yield the regularities. That is most clearly seen in the ADT version, but it holds in primitivism as well. Once we realise that laws govern worldly things through their properties, a fundamental question naturally comes to mind: how do laws 'tell' properties what to do? Differently put, why are certain properties apt to be related by nomic relations? A deep answer to the Inference Problem requires a prior answer to this question. For only given that properties are 'told' what to do by the nomic relation, can we proceed to metaphysically explain how the behaviour of particulars characterised by the thus related properties can be determined (or, in other words, how actual regularities are generated). We call the difficulty related to the above question the Governing Problem for non-Humean laws. To see the Governing Problem in action, let us look briefly at the Maxwell-Faraday law of induction $\nabla \times \mathrm{E}=-\partial \mathrm{B} / \partial \mathrm{t}$ ( $\mathrm{E}$ and $\mathrm{B}$ are the electric field strength and the magnetic field strength, respectively). On this law, a time-varying magnetic field will always accompany a spatially varying electric field, and vice versa. A solution to the Governing Problem requires an answer to the following question: how does the Maxwell-Faraday law 'tell' E and B (construed as properties of the electromagnetic field at each point) what to do? After we have metaphysically explained how the nomic relation embodied in the Maxwell-Faraday law manages to determine how $\mathrm{E}$ and $\mathrm{B}$ behave, that we can proceed to metaphysically explain how the Maxwell-Faraday law can determine that in any electric generator, the varying magnetic field created by a rotating bar magnet invariably accompanies an electric field in a nearby wire.

\subsection{ADT-laws vs Primitive Laws}

How do extant versions of LP fare with solving the Governing Problem? As far as ADT theories are concerned, no satisfactory solution has been offered. Any attempt at a solution is a 'brute fact' one. The law-making property $\mathrm{N}$ is contingent; it just happens to obtain and to relate these and not other properties. It should be clear that a necessary step for a fair solution of the Governing Problem requires an ontic account of the nomic necessitation relation. ADT theorists have either tried to identify $\mathrm{N}$ (for example Armstrong $(1993,422)$ suggested that is actually the causal relation at the type-level) or to posit it as a theoretical entity that fulfils a specific nomic role (Tooley 1987). Each of these options, however, faces its own problems. For instance, as regards the first option, it is not clear whether Armstrong's identification accommodates all kinds of laws (are all laws causal?) or even whether the entity with which the identification is made exists or not (does causation exist at the fundamental level or is it a 'folk' derivative notion?). While the second option faces the problem of multiple realisation of the definitional role by different entities.

A different kind of problem that besets the ADT conception of laws is that it is by and large qualitative. Although one might point out that the core idea behind ADT is certainly science-informed, as laws on this view are relations between natural properties, the theory is often presented by simple toy-examples rather than actual

\footnotetext{
${ }^{8}$ It is important to note that there exist strong reasons to think that the Inference Problem equally besets those versions of PL according to which the nature of modal properties is relationally constituted by second-order stimulus-response relations. As Barker and Smart (2012) argue, on those versions of PL what does the governing is not the first-order instantiations of properties but the aforementioned second-order relational facts. In that case, however, PL's explanation of governing does not significantly differ from Armstrong's account of governing in terms of the nomic necessitation relation and therefore PL inherits the Inference Problem from LP.
} 
scientific laws which are typically complex and in mathematical and quantitative form. To be sure, Armstrong (1983) has made an effort to bring his account nearer to actual scientific cases. Yet, it is not clear whether ADT (based, as it is, on a few kinds of nomic relations-nomic necessitation, nomic probabilification, and perhaps nomic exclusion) can capture the complexity of scientific laws.

How about primitivism then? Does it offer a solution for the Governing Problem? Primitivism about laws takes it that laws are real, but ultimate and primitive constituents of reality, which hold with irreducible necessity and guide the kinematical and dynamical behaviour of worldly entities (Carroll 1994; Lange 2009; Maudlin 2007) ${ }^{9}$. So, primitivism tries to solve the Governing Problem by taking the modal force of laws, and thus their governing role, as given. But, by insisting that laws are sui generis entities, primitivism fails to give an illuminating account about how laws 'guide' the behaviour of worldly things. Carroll $(1987,267)$, for instance, denies that lawstatements have truth-makers, and refrains from any further analysis of the supposed facts (either nomic or non-nomic) that are the truth-makers of laws. All he says is the following: $\mathrm{P}$ is a law of nature if and only if $\mathrm{P}$ is a regularity that holds because of nature. Hence, "[l] awhood requires that nature itself-understood as distinct from anything in nature or the absence of something from nature-make the regularity true". That, we submit, is totally unilluminating. For one, all laws are 'made true' by nature. But which laws and why are made true thus? For another, Armstrong once said that we must admit the existence of nomic necessity "in the spirit of natural piety" $(1983,92)$. Yet, he went on to say a lot more by way of analysis. Carroll's treatment of nomic necessity as primitive just calls us to be pious, without even trying to explain to us why we should. ${ }^{10}$

The Governing Problem is then a challenge inherent in all versions of LP, and is extremely important from a metaphysical point of view: LP seems unable to provide a metaphysical account of how and why properties are governed (in the sense explained

\footnotetext{
${ }^{9}$ Marc Lange (2009) has developed the view that laws are maximal sets of principles which are invariantunder-counterfactual-interventions, but since he takes counterfactuals as having a primitive modal force, his view could be taken as a variant of primitivism.

10 Ontic structuralists offer yet another version of LP. Structuralism reconceptualises laws as being the ultimate structural features of reality grounded in determinable relations, where relations are understood as not being dependent on the first-order properties of objects (Ladyman \& Ross 2007; French 2014). Ontic structuralism then does away with properties as fundamental and recovers them from relations. In particular, properties are recovered from the relations embodied in the laws. Objects too, insofar as they are allowed in some sense, depend on laws. As French sums it up: "the purported objects are dependent on the structures (and here the role of symmetries in presenting that dependence is fundamental) and the properties are dependent on the laws themselves" $(2014,302)$. The relation of metaphysical dependence that French favours, is the relation between determinable and determinates: “(...) the nature of the dependence between the structure and kinds, properties, and putative 'objects' (e.g. elementary particles) is shaped, or fleshed out, by the relationship between determinables and determinates" $(2014,290)$. Laws themselves are "relation-determinables" $(2014,283)$ and as such they yield "determinate instances of these properties". We are not going to discuss here the idea for laws as relation-determinables (for reasons to be skeptical with this view see Psillos (2016)). For our purposes, the main question is whether there is conceptual space within structuralism for a notion of 'governing'. For Cei \& French, for example, in structuralism the 'governing metaphor' is replaced by the relation of metaphysical dependence. But in view of such a dependence, it does not seem to be meaningful to ask how the structure governs the properties. And yet, Cei and French admit that "there is still a kind of governance (...) in that the behaviour of entities, both unobservable, such as electrons, and observable such as chairs, can be thought of as 'governed' by the relevant laws" $(2010,37)$. As the resulting view of the structuralist account of laws is far from clear, and in particular as it is not clear whether structuralists adopt a genuine concept of governing (given their thesis of metaphysical dependence of entities and properties on the structure), we will not discuss this view further here.
} 
earlier) by laws. It seems that without at least some 'thin' power to be nomically governed, no properties can actually have a causal/nomic role and consequently particular things seem really powerless to follow any laws. Our view, then, is that we can offer a robust solution to the Governing Problem by admitting that a) properties have generic, but ontologically 'thin', powers to be related by nomic relations; and b) nomic relations are relata-specific.

In the next section we will develop such a view, which we call the Dualist Model (henceforth, DM). In advancing DM, both kinds of problems faced by PL and LP, i.e. the difficulties of reconciling lawless ontologies with central features of current physical theory and the inability of powerless ontologies to give a satisfactory account of the governing role of laws of nature, will be solved by taking the radical stance that our scientific ontology should be both lawful and powerful. But this solution requires that scientific ontology rests on two independent pillars: both laws and powers feature as distinct ontological items.

\section{A Unified Model of Laws and Powers}

As noted already, DM is directly motivated by the problems faced by PL and LP outlined above. Our key idea is that natural necessity has a double source: both governing laws and minimally powerful properties are responsible for the natural necessity and the order that characterise the phenomena of our world: governing laws require taking properties to be (in a sense to be specified) minimally powerful (and hence governable), while powers require laws to have their quantitative relations fixed.

\subsection{Thick vs Thin Powers}

Why are laws required, even though there are powers? We have already argued that insofar as there are conserved quantities in the worldly phenomena, they are governed by conservation laws, which cannot be reduced to the powers of natural substances. And exactly the same holds for symmetries in the world.

But there is another reason why we need laws, even if we assume powers: powers, on their own, cannot fix the functional relations among properties. Friends of powers just assume that the powers properties have are fully specific, viz., they fix the exact quantitative relations among properties. But there is no good reason to think like this. To fix our ideas, let us think of mass in the classical-Newtonian world. Newton showed that gravitational force depends on (is a function of) mass and distance: it's proportional to the masses of the gravitating bodies and inversely proportional to their distance. In other words, Newton showed that a piece of matter has a power to attract another piece of matter in relation to its mass. We could then say that mass has the power to attract mass. But why should we assume that this power is such that it yields, necessarily, the exact relation of inverse-square of the distance $r$ between two masses? Would the attractive power of mass be different had the inverse-square-of-the-distance relation been different? It seems perfectly OK to say that mass would still have the power to attract other masses, had the relation been inversely proportional to some other power of the distance. In fact, this is evinced by the case of the anomalous perihelion of Mercury. Asaph Hall, in 1894, had suggested that a replacement of $1 / \mathrm{r}^{2}$ with $1 / \mathrm{r}^{2+\lambda}$, where $\lambda$ stands for a small number, was sufficient to explain the anomalous perihelion motion of Mercury. But this revision led to conflicts with other phenomena, such as the position of the Lunar apogee. The point here is simply that had this revision been correct, that is, had the law-like connection between gravitational force and masses been different, masses would still have the power to attract each other. What 
would have changed would have been the exact quantitative relation among the relevant properties.

In light of this, we may then discern two possible grades of modal strength of mass:

- Necessarily, mass confers on the objects that possess it the disposition to attract other massive objects with the exact mathematical form of attraction determined by Newton's gravitation law.

- Necessarily, mass confers on the objects that possess it the disposition to attract other massive objects.

The difference between the two grades is very important. Let us, for simplicity, refer to the power of mass (as opposed to the powers of massive particulars) and note that the two grades above attribute different powers to mass. On the first option, the attractive power of mass is fully specific; the power is such that the functional relationship that masses enter into with other masses is fully determined by the power. This, roughly put, is the view of those who think that laws 'flow' from powers. Let's call these powers thick. On the second option, mass has indeed a power, but it is thin. It's not enough on its own to fix the quantitative relations it stands to other masses. What is also required for this is laws and specific (contingent) nomic relations. Hence on the second option mass has a thin power which in tandem with a nomic relation (e.g., the inverse-square-of-the-distance relation in the actual world) attracts other masses with an exact mathematical form of attraction determined by the contingent nomic web of each possible world in which it exists. To put the point with a bit more precision, mass confers on the objects that possess it the generic disposition to attract other massive objects but the exact functional/mathematical form of the relation to which massive objects stand (i.e., Newton's gravitation law) is determined by a web of laws.

The first option above, which is adopted by PL-ists, is, we think, wrong-headed. If mass had such a thick power, it would by itself confer the completely determined disposition of attraction on the massive objects and Newton's law would be metaphysically redundant. And though this might sound appealing to some friends of powers, we have already seen that such a view is untenable given a) the problems with deriving the functional laws from the powers of individual objects, and $b$ ) the fact that the all-important conservation laws cannot be 'flowing' out of powers of individual objects. Indeed, in Newton's case, the inverse-square-of-the-distance relation does not 'flow' directly from the power of mass to attract other masses. More is needed; for instance, the law for the force in uniform circular motion as well as Newton's third law.

Why do we take it that the inverse-square-of-the-distance relation is itself a nomic relation in which masses enter? It is a nomic relation because it is fixed by other laws. In the case of Newtonian gravity, the inverse-square-of-the-distance relation is fixed by the three laws of motion together with Kepler's harmonic law. In a possible world in which Newton's laws of motion do not hold, the centripetal forces would have different measures. Similarly, in a world in which Newton's three laws hold, but where the harmonic law didn't, centripetal forces would have different measures; hence masses would not stand in the inverse-square-of-the-distance relation with each other and with the centripetal force. It is our contention that gravity satisfies Newton's law in the specific quantitative way it does in virtue of a) the generic power mass has to attract other masses and b) the inverse-square-of-the-distance nomic relation. DM, then, adopts the second option, above. 
Now, some care is needed here. For the second option itself (if construed as the negation of the view that powers are thick) bifurcates into two versions: let's call them 'light' and 'ultra-light'. The ultra-light version would have it that properties are powerful only in the sense of having the power of being governable by a law (any law). When it comes to Newton's law, for instance, it might be that the power mass has is simply its receptivity to be governed by (Newton's) law. On this view, mass (alongside other properties) is apt to be law-governed and that's where anything powerful in it ends. All the rest is done by laws.

On the 'light' version, the powers properties have are still thin (they don't fix the laws) but quite substantive. On this view, for instance, mass is a powerful property, though minimally so; i.e. it does confer on objects possessing it a generic 'attractability', even though it cannot by itself confer on the object a fully determined and quantified disposition of attraction. Both of these options need exploring. In the sequel, we shall adopt the latter and explore it (hence, we shall leave exploring the ultra-light option for some other occasion).

According, then, to DM, and given the light version above, nomic relations are indispensable parts of the complete grounds of the lawful behaviour of objects. We take such a view to offer the most satisfactory account of the particular example of gravity in Newton's theory, and to be motivated by the problems we have seen with the monistic views.

\subsection{Thin Powers and Nomic Relations}

But why are powers required, even though there are laws and nomic relations? Part of our point is dialectical: if you start out as a dispositionalist, then we have provided what we see as strong reasons to think that powers are not enough to fix the nomological order in the world; and hence, to accept further elements-what we called nomic relations - in your ontology which contribute indispensably to fixing the laws. By the same token, if you start out as a primitivist or an ADT-theorist, that is, if you reject powers as an element in your ontology while thinking that laws govern properties and their instances, then there are strong reasons to accept the view that properties are, in some sense, powerful. Hence, we claim that properties should be taken to be (in a thin sense) powerful, for otherwise laws cannot play their governing role. ${ }^{11}$ To remind the reader, we argued in section 4 that none of the main versions of LP has the resources to address the Governing Problem. The primitivists decline to answer the question of what makes certain properties apt to be related nomically by specific nomic relations; but then their general account of laws remains unilluminating. ADT-theorists, by taking properties to be amodal, make it difficult to understand how they can be governed by laws at all; and this is the reason why it is hard to understand how ADT-laws issue in the actual regularities of the world. Thus, we take it that commitment to properties having some modal force is a necessary condition for a solution to the Governing Problem.

What would be a sufficient condition? Recall that minimally powerful properties enter into nomic relations and this constitutes laws that govern the relevant powers and their instances. The governing can be thought of (conceptually) as a two-step process. First, by being powerful (even if minimally), properties, necessarily, enter into

11 The view that powers are thin is compatible with various accounts about the nature of properties; for example, properties can be pure (thin) powers or powerful qualities. In adopting DM, we are not committed to any such specific view about properties, as such a view would require independent arguments that we leave for another occasion. 
nomic relations that are consistent with their powers. It is a fact about the actual world that some properties tend to go together while others do not. Positing minimal powers explains why certain properties are apt to be related by specific nomic relations: it is because properties have minimal powers that they tend to go together. Hence, properties are governable by laws because, due to their thin powers, they tend to enter into specific nomic relations. But that's not enough for governing, since the nomic relation, which relates the thin powers, is external to its relata. How then do nomic relations 'tell' properties what to do? The second step then is to think of nomic relations themselves as relata-specific: nomic relations relate specific properties ${ }^{12}$. Hence, nomic relations are second-order relata-specific relations. For instance, the nomic relation associated with Newton's gravitational law links two masses, the force acting on each of them and their spatial distance (and cannot link other properties/relations). Newton's law governs the behaviour of mass through this relata-specific second-order relation. Of course, focusing on only one nomic relation is an oversimplification. In fact, since properties are usually relata of a number of different nomic relations, it is the network of relata-specific nomic relations constituting the causal/nomic role of the property that governs its behaviour; nothing metaphysically more informative could be said about this governing. Simply put, to nomically govern a property (qua a thin power) is simply to determine the specific form of the second-order relata-specific relations that this property bears to other properties ${ }^{13}$.

Here is an objection to this point. Consider Coulomb's law. It is a force law, which, like the gravitation law, embodies an inverse-square-of-the-distance relation but it relates charges instead of masses. Intuitively, the 'inverse-square-of-the-distance' nomic relation is the same in the two laws, the only difference being that the relata are different. Do we want to say that these two laws embody the same nomic relation with different relata? In that case, however, nomic relations cannot be relata-specific.

This objection presupposes that nomic relations should be metaphysically construed as 'function-like' entities with 'slots' to be filled by properties. So for instance, the kind 'inverse-square-of-the-distance' nomic relation has the form $F=(\operatorname{slot} 1) \cdot(\operatorname{slot} 2) / \mathrm{r}^{2}$ where specific properties (masses, charges) are substituted for the slots. There are two ways to reply to this objection. The first is to note that even if we granted the existence of slots, it would still not follow that the slots would necessarily be undifferentiated; some of them could be 'sensitive' to the nature of the properties that can fill them. So, Newton's gravitational law and Coulomb's electrostatic law are both inverse-square dynamical laws (they have the same functional form), but, nevertheless, we could say that the nomic relations associated with those two laws were different. This, in turn, means that a nomic relation is inter alia sensitive to the nature of its relata and that fact is revealed by its relata-specificity. In effect, this answer rejects the foregoing intuition:

\footnotetext{
12 As Weiland and Betti (2008) note, the relata-specificity of first-order relations can explain the unity that characterises first-order states of affairs. Perhaps then the relata-specificity of second-order nomic relations can explain the unity of nomic facts.

${ }^{13}$ In order to avoid misunderstandings, we should make clear that according to the dualist model (whose main tenets we outline here) laws do not govern the behaviour of properties via their [i.e. properties'] powers. If that were the case, then the proposed model would not be explanatorily superior to LP theories regarding the Governing Problem because it (like LP accounts) would also invite the question of how laws manage to govern properties which, in this case, are or have (thin) powers. In our view, however, laws govern properties by having as ontological constituents relata-specific nomic relations. Nevertheless, thin powers are needed to ensure properties' nomic governability, that is, properties' capability to enter into nomic relations.
} 
laws which involve a nomic relation with the same functional form are different if the relata are different.

Alternatively, we could modify the criterion of relata-specificity in order to respect the foregoing intuition, viz. that the 'inverse-square-of-the-distance' nomic relation is the same in the two laws, the only difference being that the relata are different. According to the original definition of relata-specificity noted above, a relation $\mathrm{R}$ is relata-specific iff it relates unique and fully specific relata as soon as it exists. According to the modified account of relata-specificity a relata-specific relation need not relate just one n-tuple of relata, but one kind of relata with a certain thin power, e.g., attractivity. If that's plausible, then the same nomic relation (e.g., the 'inverse-square-of-the-distance' relation) can relate distinct properties with the same thin power (e.g., masses with masses and charges with charges). A plausible view about the 'relata-specificity' of a nomic relation is that it concerns some specific thin power of the related properties. Mass, for instance, may have the power to attract mass. If that is true, then mass can enter into relations with other masses in such a way that its attractive power is manifested. But since charges have also the power to attract other charges, there is no reason not to suppose that the actual nomic relation which, contingently, relates masses with distance and force is the same as the relation which, contingently, relates charges with distance and force.

We don't have to commit ourselves to one of the two replies to the slot objection, because for us the important point is that whatever view is adopted the laws do not become metaphysically necessary. Whichever way we think of relata-specificity, if a relation is relata-specific and holds between $P$ and $Q$ in the actual world, it holds between $\mathrm{P}$ and $\mathrm{Q}$ in all possible worlds in which the relation exists, but not necessarily in any possible world in which $\mathrm{P}$ and $\mathrm{Q}$ exist, or in any possible world whatsoever. The relata of a relata-specific relation exist independently of the relation. So, the relata need not be related by the specific nomic relation. That is, there are possible worlds in which the relata exist yet the nomic relation does not. Hence, nomic relations, qua relataspecific, are metaphysically contingent. Hence, the laws are metaphysically contingent. But at the same time, the relata, having certain thin powers, can enter into certain kinds of relations. Consider, for instance, the second-order fact that is Newton's gravitation law. This fact, in the actual world, involves the property of mass and the inverse-squareof-the-distance nomic relation. Given that mass has only a thin power, masses could relate to each other by different nomic relations; but given that mass does have this minimal power to attract other masses, they would necessarily be related by a certain 'attractive' kind of relation. The nomic fact that is Newton's gravitation law must involve a nomic relation between specific thin powerful properties. In a different possible world, the nomic relation (the inverse-square-of-the-distance relation) could be different, but given that it involves masses in the actual world, and masses have minimal power, the law would be an attractive one.

We may summarise our account in a series of short answers to four important questions.

First, how do laws govern properties?

Answer: a law governs a specific property by having a constituent-a nomic relation-which determines the specific form of the second-order relation that this property bears to other properties. 
Second, why does a nomic relation relate (and hence govern) the properties that it actually relates and not others?

Answer: because it is by its own nature relata-specific.

Third, why should properties have (or, be) thin powers?

Answer: because they must have a minimal modal nature in order to be apt to be related by nomic relations.

Fourth, why should one accept DM given that it is less parsimonious compared to monistic views such as dispositionalism or primitivism?

Answer: while parsimony is certainly a virtue in metaphysics, less parsimonious views are to be preferred to the extent that they solve problems that more parsimonious alternatives are not able to solve. We have argued that this is in fact the case for all main monistic accounts that accept either laws or powers, but not both, as fundamental elements in ontology. So, while less parsimonious, the richer ontology of DM enables it to offer an account of the ontology of laws that, we claim, it is more consonant with the scientific image as revealed by current science, and the metaphysical picture that underpins it, than any of its monistic rivals.

This, then, is in broad outline the metaphysically deflationary view we associate with DM. ${ }^{14}$ It is beyond the scope of this paper to develop a complete account of laws of nature as understood by DM. The important point to note here is that, in contrast to current monistic views about laws (such as PL and LP), we take it that the very idea that laws govern worldly regularities requires a commitment to both laws and powers as distinct and irreducible elements, which indispensably contribute to fixing the nomological structure of the world. According to DM, the specific behaviours of things in the world are the outcome of both the thin powers things have to be subjected to laws, and certain nomic features of the world (including symmetries and conservation laws). So the motto of DM is: no laws and (thin) powers in, no (governing) laws out.

\section{Other Accounts that Combine Powers and Laws}

DM is not the only theoretical proposal that tries to combine modal properties and laws. In order to further strengthen the plausibility of DM, let us in this last section examine some such recent accounts. Since our focus is on governing laws we will not discuss those views that suggest that we can combine realism about modal properties with versions of regularity theory of non-governing laws (see Vetter $(2015,289-290)$ and Demarest (2017)).

Kistler (forthcoming) thinks that powers and laws together play the metaphysical role of making true disposition attributions. He takes it that the justification of his view is that it offers the best way to make sense of what science says about the dispositions of objects. In particular, Kistler argues that laws are indispensable in making sense of what science tells us about dispositions for two reasons: first, laws are required to explain the structure of the dispositions corresponding to one power and to make sense of the systematic relations between the dispositions of different powers. And second, laws provide the relational part of the truthmaker of a disposition ascription which

14 The suggested account is metaphysically deflationary in the sense that it does not posit a sui generis governing relation. It is important to stress that the discussion concerns the governing of the behaviour of properties, not of objects. The latter is intimately related to (or even identified with) the relation of necessitation of natural regularities by laws (see, for example, Hildebrand 2013). 
cannot be offered by monadic powers. For Kistler, laws must be ontologically robust for them to be truthmakers. One way to fulfil this requirement is to think of laws (as Kistler does) as B-internal relations between properties, ${ }^{15}$ that is, internal relations whose relata are partly or wholly constituted by their entering into these relations.

Kistler's account seems to be akin to the dualist model since both powers and laws are required to metaphysically explain the dispositions of objects. Yet, secunda facie, this is not the case because Kistler adopts neutral monism about properties; that is, he thinks that the categorical/dispositional distinction is not ontological and refers to different ways of conceiving and/or describing natural properties. The term 'power' refers according to Kistler to any property that is a partial truthmaker for a disposition ascription.

Kistler's neutral monism is the basis for our main worry about his position. It is true that according to both his view and the dualist model natural properties and laws are needed to explain the order that characterises the actual world. Our model, however, can metaphysically explain the need for laws; since natural properties are by their nature modally too thin to do the explanatory job by themselves, laws are required to provide the 'missing' modality. We cannot see, however, how Kistler's account can metaphysically explain the indispensability of laws. In line with his neutral monism, he cannot say that natural properties have a dispositional or categorical or 'mixed' nature. Hence, it is an ontological brute fact ${ }^{16}$ that they are only partial truthmakers of the disposition ascriptions. Scientific practice shows that laws are an indispensable element of any adequate explanation of the behaviour and the dispositions of objects, but there is nothing in Kistler's neutral monistic account that could metaphysically explain that.

In contrast to Kistler, Tugby holds that properties have a powerful nature. In his (2016), he defends the indispensability of governing laws in the framework of a powerbased ontology. According to Tugby, the motivation to discuss seriously the role of substantial nomic relations in a powers ontology is that the relational-constitution view about powers is structurally similar to the ADT view as far as the governance of the behaviour of concrete things is concerned. More precisely, in both views second-order relations between property types help to explain the behaviour of propertied things. Of course, the difference is that the second-order relations between power types are internal and necessary, whereas ADT nomic relations are external and contingent. Yet, the latter fact would have an impact on the similarity between the views only provided that the nomic relations between power types, qua internal, were no additions of being. Tugby argues, however, that qua B-internal, nomic relations are metaphysically robust $(2016,1152)$. So, in Tugby's view, power theorists face their own inference problem and in order to solve it they have to investigate the role of second-order nomic relations between powers in the governance of concrete things.

Tugby argues that power types are Platonic entities which are essentially constituted by B-internal second-order nomic relations. The latter, though necessary and internal, are external to the concrete things because a) qua Platonic are capable of uninstantiated existence, and b) the first order states of affairs which are their instances ontologically depend on them and not vice versa. For Tugby, this kind of externality is a necessary condition for these relations to govern. Now, power types, though capable of uninstantiated existence, are often instantiated by concrete things. Tugby defends an

15 Here we follow Barker's (2009) terminology for the different kinds of internal relations.

${ }^{16}$ Kistler argues that since the ascription of a disposition is a relational fact, its truthmaker should have a relational part (which, for him, is the law). The ontological structure of a truthmaker, however, does not have to mirror the structure of its associated truth. 
account of instantiation as a kind of ontological dependence; in particular, as a nonspatial constituency relation. Hence, for him, power instances are first-order states of affairs which have as essential non-spatial constituents their associated power types $(2016,1160)$. The latter have independent existence, but they can still be constituents of their instances.

Given all that, the 'inference problem' for powers is 'solved' because: a) power types can be multiply instantiated and, so, many things can have as a constituent the same power type and consequently have the same dispositions $(2016,1157)$, and b) power instances (due to their ontological dependence on the associated power types) inherit their 'directedness' for specific manifestations from the relations their power types bear $(2016,1158)$.

Compared to our view, Tugby's notion of governance concerns the behaviour of concrete things and consequently it is related to the 'traditional' inference problem. Our Governing Problem, however, concerns the behaviour of properties (that is, the determination of their nomic/causal role). Tugby does not examine the Governing Problem as we understand it, but given what he says about the inference problem it is natural to think that his 'solution' to our Governing Problem would be that properties are thick powers whose very essence includes the nomic relations. Without taking into account the various difficulties Platonism in general faces, Tugby's approach to the Governing Problem would be explanatorily adequate provided that all nomic relations can somehow be associated with the essences of particular powers. But if our previous arguments are sound, this is not true.

Let us finally focus on the views recently presented by Dumsday (2019). Dumsday (2019) offers the following argument for the view that realism about modal properties implies nomic realism (he calls it Nomic Dispositionalism):

Premise 1 At least some dispositions have ceteris paribus (cp) clauses incorporating uninstantiated universals (which cp clauses help to delimit the range of manifestations of those dispositions).

Premise 2 If at least some dispositions have cp clauses incorporating uninstantiated universals (which cp clauses help to delimit the range of manifestations of those dispositions), then laws of nature exist.

Conclusion Therefore laws of nature exist. $(2019,11-12)$

The basic idea behind Dumsday's argument (expressed in premise 2) is that for the realist about modal properties, cp clauses that involve uninstantiated universals are (better, express) laws. Hence, if the realist about modal properties needs to posit $\mathrm{cp}$ clauses, then the conclusion follows: there exist governing laws.

More precisely: the identity conditions of dispositions associated with modal properties consist in their stimulus and manifestation conditions, including any cp clauses. At least some dispositions, however, have cp clauses that include uninstantiated universals in the sense that they include either uninstantiated values of an instantiated type of universal or 'alien' properties never found instantiated in our world but whose instantiation is possible. ${ }^{17}$ So, the uninstantiated universals (pure abstracta) figuring in

17 What determines this possibility? The dispositional nature of modal properties or independent laws? What kind of possibility is this? If it is physical, we probably have a circularity here. Alien properties should be physically possible in order to figure in cp clauses but the latter delimit the range of disposition-manifestation and so, according to power realists' own lights, determine the physically possible events. 
cp clauses help to delimit the range of possible disposition-manifestations and function as (at least partial) truthmakers for various counterfactuals concerning the manifestations of those dispositions. Therefore, they contribute to the fact that events in our world must take place in certain ways rather than others. Dumsday $(2019,14)$ explains: "If an uninstantiated value of positive charge were instantiated in entities possessing mass, then where those entities would normally undergo a gravitational attraction of a certain force, they might instead be repelled. Or if an alien universal were instantiated, the 'normal' disposition manifestation might otherwise be disrupted. Even in their uninstantiated state, these universals serve as truthmakers for counterfactuals involving actual, instantiated dispositions. This counts as playing a governing role in the physical universe, in the relevant sense...". Cp clauses then 'include' abstracta determining that certain events can or cannot take place under particular circumstances. But for Dumsday laws are properly conceived as abstract entities somehow playing a governing role in the physical universe. Hence, cp clauses (by incorporating abstracta that do exactly that) should be laws.

There is a number of objections about nomic dispositionalism (ND), some of which Dumsday himself is at pains to meet. The first objection is that the account of laws given by ND is divorced from typical scientific usage. According to ND, laws of nature are not what scientists typically call laws but are instead the cp clauses figuring in the identity conditions of those modal properties that appear in scientific laws. Dumsday $(2019,21)$ admits that this a disadvantage for his view but points out that ND is closer to scientific use of law-talk than traditional realism about modal properties because in contrast to the latter it allows some robust governing laws to exist. This response is hardly adequate because the problem is not whether ND is better than traditional realism about modal properties but rather whether cp clauses can be regarded by scientists as law-statements (we take it that Dumsday view is that cp clauses are law statements that correspond to abstract entities that govern). For scientists, however, the typical expression of a law-statement is a functional relation between physical properties and not a cp clause. So, if we want to have a metaphysics of laws that is friendly to scientific practice (as Dumsday advertises his view), we should refrain from adopting views which are so remote from what scientists believe that actual laws are. This is then a main difference between ND and the dualist model that strongly counts in favour on the latter, since in DM, governing laws have exactly the form of laws as revealed by science.

A second objection against ND is that cp clauses cannot be governing laws because they cannot govern the behaviour of worldly things. The reason for being unable to govern is that cp clauses are existentially dependent on their associated modal properties of things and nothing seems to be able to govern what is existentially dependent on. The existential dependence of cp clauses is due to the fact that they are parts of the identity conditions of dispositions associated with modal properties of things $(2019,15)$. Dumsday's response is that the uninstantiated universals figuring in a cp clause associated with a modal property cannot be literally constituents of an instance of that property. For him, what is really happening is that an instance of a modal property has an extrinsic necessary relation to these universals and that does not rule out that one relatum of the relation can govern the other one.

It is not clear, however, whether there can exist a governing relation between different levels (the type-level of uninstantiated universals and the token-level of property-instance) that is not the instantiation relation (if the latter exists). And even if we skip this difficulty, it seems that Dumsday's response fails to capture what the objection really is. For cp clauses are involved in the identity conditions of property- 
types and therefore the objector does not claim that their constituents (that is, the uninstantiated universals) are literally parts of property-tokens. The problem of course remains in the case of modal property-types whose identity involves alien universals. How can the identity of a modal property-type be determined by relations to universals with no instantiations at all? Dumsday tracks the conceptual space of possible immanent-universalism-friendly solutions to the problem albeit with intention to meet the above-mentioned independence objection. But he admits that the simplest response to the problem is to embrace platonic realism for modal properties. However, such commitment to platonic realism about universals is not required in the case of the dualist model, which can remain neutral about the metaphysics of universals. But then, DM is ontologically more minimal than ND (as well as Tugby's Platonic Dispositionalism mentioned earlier), and thus to be preferred.

A final objection is that ND relies on the assumption that the identity of modal properties depends on cp clauses. But as Tugby $(2016,1149)$ points out, some philosophers such as Bird $(2007,60-65)$ have argued that the identity conditions of fundamental properties do not involve cp clauses because it is impossible (or at least implausible to suppose) that there are interfering factors at the fundamental level. Hence, if at least some modal properties are fundamental or if at least some laws relate fundamental modal properties, then ND fails to provide an adequate metaphysical account of all laws.

\section{Conclusions}

We have argued that neo-Aristotelian accounts that take powers to be the sole source of natural necessity and nomological order in the world face fundamental difficulties, stemming from their inability to account for central features of scientific practice and theorising. In particular, we have shown that powerful but lawless ontologies have trouble accounting for functional laws among quantities, conservation laws, symmetries, as well as the role of counterlegals in scientific practice. We have thus identified a fundamental limitation of neo-Aristotelianism, in providing an ontology based solely on the powers of worldly entities that is compatible with current science. However, we have also seen that alternative lawful but powerless ontologies also face a central difficulty in clarifying how laws can govern worldly entities.

We have outlined and defended a new approach — the dualist model—which, we have argued, is a novel and promising candidate for providing an ontological picture that is in conformity with current science but at the same time metaphysically illuminating. In this dualist model, both categories (laws and powers, suitably conceived) are equally fundamental and irreducible to each other. So, DM rejects an implicit presupposition in most current philosophical discussions in the metaphysics of science, i.e. that the categories of laws and powers are mutually exclusive and hence that a metaphysics of nature has to be some version of monism and take the form either of PL or of LP.

While more needs to be said for a full articulation of the ontology associated with the dualist model (which we leave for future work), we think we have done enough in this paper to motivate DM and show why it is a very promising candidate for providing a scientific ontology that is as close to current science as possible. To sum up, the general picture emerging from DM can be put as follows: the worldly things execute the laws via their thin causal powers, whilst the laws determine the quantitative relations between the properties of worldly things, the temporal evolution of their states and their interactions, as well as global features of the world (e.g. conservation principles). 
DM offers a third way-a via media-which recommends an ontology that is both lawful and powerful. Our claim has been that such an ontology has the resources to solve the difficulties encountered by its two dominant competitors, without inheriting the problems of either of them. Developing a metaphysically adequate account of how laws govern worldly entities provides thus a good example of how metaphysical views can have a useful role in our attempts to offer philosophically satisfactory accounts of the current scientific image.

\section{References}

Armstrong, D.M. (1983). What is a Law of Nature? Cambridge: Cambridge University Press.

Armstrong, D.M. (1993). The Identification Problem and the Inference Problem. Philosophy and Phenomenological Research 53, 421-422.

Barker, S. (2009). Dispositional Monism, Relational Constitution and Quiddities. Analysis 69(2), 242-250.

Barker, S. and Smart, B. (2012). The Ultimate Argument Against Dispositional Monist Accounts of Laws. Analysis 72(4), 714-722.

Bigelow, J., Ellis, B. \& Lierse, C. (1992). The World as One of a Kind: Natural Necessity and Laws of Nature. British Journal for the Philosophy of Science 43, 371-88.

Bird, A. (2007). Nature's Metaphysics: Laws and Properties. Oxford: Oxford University Press.

Carroll, J.W. (1994). Laws of Nature. Cambridge: Cambridge University Press. Cartwright, N. \& Ward, K. (eds.) (2016). Rethinking Order: After the Laws of Nature. London: Bloomsbury.

Cartwright, N. and Pemberton, J. (2013) Aristotelian Powers: Without Them, What Would Modern Science Do? In Groff, R. and Greco, J. (eds.) Powers and capacities in philosophy: the new Aristotelianism. New York: Routledge, 93-112.

Cei, A. \& French, S. (2010). Getting Away from Governance: A Structuralistic Approach to Laws and Symmetries. Methode: Analytic Perspectives 4, 25-48.

Chakravartty, A. (2019). Physics, Metaphysics, Dispositions, and Symmetries - À la French. Studies in History and Philosophy of Science 74, 10-15.

Demarest, H. (2017). Powerful Properties, Powerless Laws. In Jacobs, J. (ed.) Causal Powers, Oxford: Oxford University Press, 38-53.

Descartes, R. (1982) [1644]. Principles of Philosophy. Miller, V.R. and Miller, R.P. (trans) Dordrecht: D. Reidel Publishing Company.

Dretske, F. (1977). Laws of Nature. Philosophy of Science 44, 248-268.

Dumsday, T. (2019). Dispositionalism and the Metaphysics of Science. Cambridge:

Cambridge University Press.

Ellis, B. (1999). Causal Powers and Laws of Nature \& Response to David Armstrong. In

Sankey, H. (ed.) Causation and Laws of Nature, Dordrecht: Kluwer Academic Publishers, 19-34, 39-44.

Ellis, B. (2001). Scientific Essentialism. Cambridge: Cambridge University Press.

Ellis, B. (2005). Universals, the Essential Problem and Categorical Properties. Ratio 18, 462-472.

Fraassen, B. V. (1989). Laws and Symmetry. Oxford: Clarendon Press.

French, S. (2014). The Structure of the World: Metaphysics and Representation. Oxford: Oxford University Press.

Handfield, T. (2004). Counterlegals and Necessary Laws. The Philosophical Quarterly 54, 402-419. 
Hildebrand, T. (2013). Tooley's Account of the Necessary Connection Between Law and Regularity. Philosophical Studies 166, 33-43.

Kistler, M. (forthcoming). Powers, Dispositions, and Laws of Nature. In Meincke, A.S. (ed) Dispositionalism: Perspectives from Metaphysics and the Philosophy of Science, Ch.11, Springer.

Ladyman, J. \& Ross, D. (2007). Every Thing Must Go. New York: Oxford University Press. Lange, M. (2009). Laws and Lawmakers: Science, Metaphysics, and the Laws of Nature. Oxford: Oxford University Press.

Livanios, V. (2010). Symmetries, Dispositions and Essences. Philosophical Studies 148, 295-305.

Livanios, V. (2014). The "Constant" Threat to the Dispositional Essentialist Conception of Laws. Metaphysica 15, 129-155.

Livanios, V. (2017). Science in Metaphysics: Exploring the Metaphysics of Properties and Laws. Cham: Palgrave Macmillan.

Livanios, V. (2018). Dispositionality and Symmetry Structures. Metaphysica 19, 201217.

Marmodoro, A. (ed.) (2010). The Metaphysics of Powers: Their Grounding and Their Manifestations. London: Routledge.

Maudlin, T. (2007). The Metaphysics within Physics, Oxford: Oxford University Press. Molnar, G. (2003). Powers: A Study in Metaphysics. Oxford: Oxford University Press. Mumford, S. (2004). Laws in Nature. London: Routledge.

Mumford, S. \& Anjum, R. L. (2011). Getting Causes from Powers, Oxford: Oxford University Press.

Mumford, S. \& Anjum, R. L. (2018). What Tends to Be: The Philosophy of Dispositional Modality. New York: Routledge.

Noether, E. (1918). Invariante Variationsprobleme. Nachr. d. Konig. Gesellschd. d.Wiss. Zu Gottingen, Math-phys. Klasse, 235-57.

Pages, J. (2002). The Dretske-Tooley-Armstrong Theory of Natural Laws and the Inference Problem. International Studies in the Philosophy of Science 16, 227-243. Psillos, S. (2016). Broken Structuralism. Metascience 25(2), 163-171.

Psillos, S. (2018). Laws and Powers in the Frame of Nature. In Patton, L. \& Ott, W. (Eds)

Laws of Nature. Oxford: Oxford University Press, 80-107.

Schaffer, J. (2016). It is the Business of Laws to Govern. Dialectica 70(4), 577-588.

Schneider, S. (2007). What is the Significance of the Intuition that Laws of Nature

Govern? Australasian Journal of Philosophy 85(2), 307-324.

Sider, T. (1992). Tooley's Solution to the Inference Problem. Philosophical Studies 67, 261-275.

Tooley, M. (1977). The Nature of Law. Canadian Journal of Philosophy 7, 667-698. Tooley, M. (1987). Causation: A Realist Approach. Oxford: Clarendon Press.

Tugby, M. (2016). Universals, Laws, and Governance. Philosophical Studies 173(5), 1147-1163.

Vetter, B. (2009). Review of Nature's Metaphysics: Laws and Properties. Logical Analysis and History of Philosophy 12, 320-328.

Vetter, B. (2012). Dispositional Essentialism and the Laws of Nature. In Bird, A., Ellis, B., and Sankey, H. (Eds) Properties, Powers and Structures: Issues in the Metaphysics of Realism. New York: Routledge, 201-215.

Vetter, B. (2015). Potentiality: From Dispositions to Modality. Oxford: Oxford University Press. 
Weiland, J. W., \& Betti, A. (2008). Relata-specific Relations: A Response to Vallicella. Dialectica 62(4), 509-524. 